Kragujevac Journal of Mathematics

Volume 44(1) (2020), PAges 127-143.

\title{
OSTROWSKI-GRÜSS TYPE INEQUALITIES AND A 2D OSTROWSKI TYPE INEQUALITY ON TIME SCALES INVOLVING A COMBINATION OF $\triangle$-INTEGRAL MEANS
}

\author{
SETH KERMAUSUOR ${ }^{1}$ AND EZE R. NWAEZE ${ }^{2 *}$
}

\begin{abstract}
In this paper, we derived two Ostrowski-Grüss type inequalities on time scales involving a combination of $\Delta$-integral means. One of the inequalities is sharp. We also obtained 2-dimensional Ostrowski type inequality involving a combination of $\Delta$-integral means. Our results extend some known results in the literature. Furthermore, we apply our results to the continuous, discrete and quantum calculus to obtain some interesting inequalities in these directions.
\end{abstract}

\section{INTRODUCTION}

In 1938, Alexander Ostrowski [23] provided a bound for the deviation of a function from its integral mean. The inequality, which is today known in the literature as Ostrowski inequality, states as follows.

Theorem 1.1. Let $f:[a, b] \rightarrow \mathbb{R}$ be continuous on $[a, b]$ and differentiable in $(a, b)$ and its derivative $f^{\prime}:(a, b) \rightarrow \mathbb{R}$ is bounded in $(a, b)$. If $\left|f^{\prime}(t)\right| \leq M$ for all $t \in[a, b]$, then we have

$$
\left|f(x)-\frac{1}{b-a} \int_{a}^{b} f(t) d t\right| \leq\left(\frac{1}{4}+\frac{\left(x-\frac{a+b}{2}\right)^{2}}{(b-a)^{2}}\right)(b-a) M,
$$

for all $x \in[a, b]$. The inequality is sharp in the sense that the constant $1 / 4$ cannot be replaced by a smaller one.

Key words and phrases. Montgomery identity, Ostrowski's inequality, Ostrowski-Grüss inequality, $\Delta$-integral means, double integrals, time scales.

2010 Mathematics Subject Classification. Primary: 35A23. Secondary: 26E70, 34N05.

DOI 10.46793/KgJMat2001.127K

Received: November 11, 2017.

Accepted: February 27, 2018. 
This inequality has received considerable attention over the past years (see for example $[9,10,19]$ and the references therein). In 1997, Dragomir and Wang [9] obtained the following Ostrowski-Grüss type integral inequality.

Theorem 1.2. Let $I \subset \mathbb{R}$ be an open interval, $a, b \in I, a<b$. If $f: I \rightarrow \mathbb{R}$ is $a$ differentiable function such that there exist constants $\gamma, \Gamma \in \mathbb{R}$, with $\gamma \leq f^{\prime}(x) \leq \Gamma$ for all $x \in[a, b]$, then we have

$$
\left|f(x)-\frac{1}{b-a} \int_{a}^{b} f(t) d t-\frac{f(b)-f(a)}{b-a}\left(x-\frac{a+b}{2}\right)\right| \leq \frac{1}{4}(b-a)(\Gamma-\gamma),
$$

for all $x \in[a, b]$.

In 1988, the German mathematician Stefan Hilger [11] introduced the theory of time scales to unify the continuous and discrete calculus in a consistent manner. Since then many authors have studied several integral inequalities on time scales for functions of a single variable (see $[15,19,22,26]$ and the references therein) as well as for functions of two independent variables (see $[12,13,17,18,24,25]$ and the references therein). In 2008, Bohner and Matthews [2] extended Theorem 1.1 to an arbitrary time scale $\mathbb{T}$ as follows.

Theorem 1.3. Let $a, b, s, t \in \mathbb{T}, a<b$ and $f:[a, b] \rightarrow \mathbb{R}$ be a differentiable. Then

$$
\left|f(t)-\frac{1}{b-a} \int_{a}^{b} f(\sigma(s)) \Delta s\right| \leq \frac{M}{b-a}\left[h_{2}(t, a)+h_{2}(t, b)\right]
$$

where $h_{2}(\cdot, \cdot)$ is defined by Definition 2.8 in Section 2 and $M=\sup _{a<t<b}\left|f^{\Delta}(t)\right|<\infty$. Inequality (1.1) is sharp in the sense that the right-hand side cannot be replaced by $a$ smaller one.

In 2009, Liu and Ngô [20] used the Grüss inequality obtained by Bohner and Matthews [2] to extend Theorem 1.2 to an arbitrary time scale as follows.

Theorem 1.4. Suppose $a, b, x, t \in \mathbb{T}$ and $f:[a, b] \rightarrow \mathbb{R}$ is differentiable. Suppose $f^{\Delta} \in C_{r d}$ and $\gamma \leq f^{\Delta}(x) \leq \Gamma$ for all $x \in[a, b]$ and some $\gamma, \Gamma \in \mathbb{R}$. Then we have

$$
\left|f(x)-\frac{1}{b-a} \int_{a}^{b} f(\sigma(t)) \Delta t-\frac{f(b)-f(a)}{(b-a)^{2}}\left(h_{2}(x, a)-h_{2}(x, b)\right)\right| \leq \frac{1}{4}(b-a)(\Gamma-\gamma),
$$

for all $x \in[a, b]$.

The same authors in [21] obtained a sharp bound for the inequality in Theorem 1.4. Specifically, they proved the next theorem.

Theorem 1.5. Suppose $a, b, x, t \in \mathbb{T}$ and $f:[a, b] \rightarrow \mathbb{R}$ is differentiable. Suppose also $f^{\Delta} \in C_{r d}$ and $\gamma \leq f^{\Delta}(x) \leq \Gamma$ for all $x \in[a, b]$ and some $\gamma, \Gamma \in \mathbb{R}$. Then we have

$$
\left|f(x)-\frac{1}{b-a} \int_{a}^{b} f(\sigma(t)) \Delta t-\frac{f(b)-f(a)}{(b-a)^{2}}\left(h_{2}(x, a)-h_{2}(x, b)\right)\right|
$$




$$
\leq \frac{\Gamma-\gamma}{2(b-a)} \int_{a}^{b}\left|K(x, t)-\frac{h_{2}(x, a)-h_{2}(x, b)}{b-a}\right| \Delta t,
$$

for all $x \in[a, b]$, where

$$
K(x, t)= \begin{cases}t-a, & a \leq t<x, \\ t-b, & x \leq t \leq b .\end{cases}
$$

Motivated by the above works and the paper [17], we obtain two Ostrowski-Grüss type inequalities on time scales involving a combination of $\Delta$-integral means. The results above then become particular case of our results. Also, we provide a 2D Ostrowski type inequality for double integrals involving a combination of $\Delta$-integral means. The result in [17] then becomes a particular case of our result.

This paper is arranged in the following order: first, we present some time scale essentials in Section 2. In Section 3, our first two results are formulated and proved. Finally, we provide a 2D Ostrowski-type inequality in Section 4.

\section{Some Basic Notions of Time Scales}

In this section, we briefly recall some fundamental facts about the time scale theory. For further details and proofs we invite the interested reader to Hilger's Ph.D. thesis [11], the books $[4,5,16]$, and the survey [1].

Definition 2.1. A time scale is an arbitrary nonempty closed subset of the real numbers $\mathbb{R}$.

Throughout this work we assume $\mathbb{T}$ is a time scale and $\mathbb{T}$ has the topology that is inherited from the standard topology on $\mathbb{R}$. It is also assumed throughout that in $\mathbb{T}$ the interval $[a, b]$ means the set $\{t \in \mathbb{T}: a \leq t \leq b\}$ for the points $a<b$ in $\mathbb{T}$. Since a time scale may not be connected, we need the following concept of jump operators.

Definition 2.2. The forward and backward jump operators $\sigma, \rho: \mathbb{T} \rightarrow \mathbb{T}$ are defined by $\sigma(t)=\inf \{s \in \mathbb{T}: s>t\}$ and $\rho(t)=\sup \{s \in \mathbb{T}: s<t\}$, respectively.

The jump operators $\sigma$ and $\rho$ allow the classification of points in $\mathbb{T}$ as follows.

Definition 2.3. If $\sigma(t)>t$, then we say that $t$ is right-scattered, while if $\rho(t)<t$ then we say that $t$ is left-scattered. Points that are right-scattered and left-scattered at the same time are called isolated. If $\sigma(t)=t$, then $t$ is called right-dense, and if $\rho(t)=t$ then $t$ is called left-dense. Points that are both right-dense and left-dense are called dense.

Definition 2.4. The graininess function $\mu: \mathbb{T} \rightarrow[0, \infty)$ is defined by $\mu(t)=\sigma(t)-t$ for $t \in \mathbb{T}$. The set $\mathbb{T}^{k}$ is defined as follows: if $\mathbb{T}$ has a left-scattered maximum $m$, then $\mathbb{T}^{k}=\mathbb{T}-\{m\}$, otherwise, $\mathbb{T}^{k}=\mathbb{T}$.

If $\mathbb{T}=\mathbb{R}$, then $\mu(t)=0$ and when $\mathbb{T}=\mathbb{Z}$, we have $\mu(t)=1$. 
Definition 2.5. Let $f: \mathbb{T} \rightarrow \mathbb{R}$ and $t \in \mathbb{T}^{k}$. Then we define $f^{\Delta}(t)$ to be the number (provided it exists) with the property that for any given $\epsilon>0$ there exists a neighborhood $U$ of $t$ such that

$$
\left|f(\sigma(t))-f(s)-f^{\Delta}(t)[\sigma(t)-s]\right| \leq \epsilon|\sigma(t)-s|, \quad \text { for all } s \in U .
$$

We call $f^{\Delta}(t)$ the delta derivative of $f$ at $t$. Moreover, we say that $f$ is delta differentiable (or in short: differentiable) on $\mathbb{T}^{k}$ provided $f^{\Delta}(t)$ exists for all $t \in \mathbb{T}^{k}$. The function $f^{\Delta}: \mathbb{T}^{k} \rightarrow \mathbb{R}$ is then called the delta derivative of $f$ on $\mathbb{T}^{k}$.

In the case $\mathbb{T}=\mathbb{R}, f^{\Delta}(t)=\frac{d f(t)}{d t}$. In the case $\mathbb{T}=\mathbb{Z}, f^{\Delta}(t)=\Delta f(t)=f(t+1)-f(t)$, which is the usual forward difference operator. If $\mathbb{T}=q^{\mathbb{N}_{0}}, q>1$ and $\mathbb{N}_{0}=\mathbb{N} \cup\{0\}$, then $f^{\Delta}(t)=\frac{f(q t)-f(t)}{(q-1) t}$.

Theorem 2.1. Assume $f, g: \mathbb{T} \rightarrow \mathbb{R}$ are differentiable at $t \in \mathbb{T}^{k}$. Then the product $f g: \mathbb{T} \rightarrow \mathbb{R}$ is differentiable at $t$ with

$$
(f g)^{\Delta}(t)=f^{\Delta}(t) g(t)+f(\sigma(t)) g^{\Delta}(t)
$$

Definition 2.6. The function $f: \mathbb{T} \rightarrow \mathbb{R}$ is said to be rd-continuous on $\mathbb{T}$ provided it is continuous at all right-dense points $t \in \mathbb{T}$ and its left-sided limits exist at all left-dense points $t \in \mathbb{T}$. The set of all rd-continuous function $f: \mathbb{T} \rightarrow \mathbb{R}$ is denoted by $C_{r d}(\mathbb{T}, \mathbb{R})$. Also, the set of functions $f: \mathbb{T} \rightarrow \mathbb{R}$ that are differentiable and whose derivative is rd-continuous is denoted by $C_{r d}^{1}(\mathbb{T}, \mathbb{R})$.

It follows from [2, Theorem 1.74] that every rd-continuous function has an antiderivative.

Definition 2.7. Let $F: \mathbb{T} \rightarrow \mathbb{R}$ be a function. Then $F: \mathbb{T} \rightarrow \mathbb{R}$ is called the antiderivative of $f$ on $\mathbb{T}$ if it satisfies $F^{\Delta}(t)=f(t)$ for any $t \in \mathbb{T}^{k}$. In this case, the Cauchy integral

$$
\int_{a}^{b} f(t) \Delta t=F(b)-F(a), \quad a, b \in \mathbb{T} .
$$

Theorem 2.2. If $a, b, c \in \mathbb{T}$ with $a<c<b, \alpha \in \mathbb{R}$ and $f, g \in C_{r d}(\mathbb{T}, \mathbb{R})$, then

(i) $\int_{a}^{b}[f(t)+g(t)] \Delta t=\int_{a}^{b} f(t) \Delta t+\int_{a}^{b} g(t) \Delta t$;

(ii) $\int_{a}^{b} \alpha f(t) \Delta t=\alpha \int_{a}^{b} f(t) \Delta t$;

(iii) $\int_{a}^{b} f(t) \Delta t=-\int_{b}^{a} f(t) \Delta t$;

(iv) $\int_{a}^{b} f(t) \Delta t=\int_{a}^{c} f(t) \Delta t+\int_{c}^{b} f(t) \Delta t$;

(v) $\left|\int_{a}^{b} f(t) \Delta t\right| \leq \int_{a}^{b}|f(t)| \Delta t$;

(vi) $\int_{a}^{b} f(t) g^{\Delta}(t) \Delta t=(f g)(b)-(f g)(a)-\int_{a}^{b} f^{\Delta}(t) g^{\sigma}(t) \Delta t$.

Definition 2.8. Let $h_{k}: \mathbb{T}^{2} \rightarrow \mathbb{R}, k \in \mathbb{N}_{0}$ be defined by $h_{0}(t, s)=1$ for all $s, t \in \mathbb{T}$ and then recursively by $h_{k+1}(t, s)=\int_{s}^{t} h_{k}(\tau, s) \Delta \tau$ for all $s, t \in \mathbb{T}$. 
If $\mathbb{T}=\mathbb{R}$, then $h_{k}(t, s)=\frac{(t-s)^{k}}{k !}$ for all $s, t \in \mathbb{R}$. If $\mathbb{T}=\mathbb{Z}$, then $h_{k}(t, s)=\left(\begin{array}{c}t-s \\ k\end{array}\right)$ for all $s, t \in \mathbb{Z}$. If $\mathbb{T}=q^{\mathbb{N}_{0}}, q>1$, then $h_{k}(t, s)=\Pi_{\nu=0}^{k-1} \frac{t-q^{\nu} s}{\sum_{\mu=0}^{\nu} q^{\mu}}$ for all $s, t \in q^{\mathbb{N}_{0}}$.

\section{Ostrowski-Grüss Type Inequality Involving a Combination of $\Delta$-INTEGRAL MEANS}

To prove our theorems, we need the following lemmas. The first lemma was first provided in [8] for the case $\mathbb{T}=\mathbb{R}$ and extended to any arbitrary time scale in [14].

Lemma 3.1 (Montgomery identity involving a combination of $\Delta$-integral means). Let $a, b, t \in \mathbb{T}, a<b$ and $f:[a, b] \rightarrow \mathbb{R}$ be differentiable. Then for all $x \in[a, b]$, we have

$$
\int_{a}^{b} P(x, t) f^{\Delta}(t) \Delta t=f(x)-\frac{1}{\alpha+\beta}\left[\frac{\alpha}{x-a} \int_{a}^{x} f(\sigma(t)) \Delta t+\frac{\beta}{b-x} \int_{x}^{b} f(\sigma(t)) \Delta t\right],
$$

where $\alpha, \beta \in \mathbb{R}$ are nonnegative and not both zero, and

$$
P(x, t)= \begin{cases}\frac{\alpha}{\alpha+\beta}\left(\frac{t-a}{x-a}\right), & a \leq t<x, \\ \frac{-\beta}{\alpha+\beta}\left(\frac{b-t}{b-x}\right), & x \leq t \leq b .\end{cases}
$$

The next lemma is the Grüss inequality on time scales obtained by Bohner and Matthews [2].

Lemma 3.2. [2] Let $a, b, s \in \mathbb{T}, f, g \in C_{r d}$ and $f, g:[a, b] \rightarrow \mathbb{R}$. Then for

$$
m_{1} \leq f(s) \leq M_{1}, \quad m_{2} \leq g(s) \leq M_{2}
$$

we have

$$
\begin{aligned}
& \left|\frac{1}{b-a} \int_{a}^{b} f^{\sigma}(s) g^{\sigma}(s) \Delta s-\frac{1}{(b-a)^{2}} \int_{a}^{b} f^{\sigma}(s) \Delta s \int_{a}^{b} g^{\sigma}(s) \Delta s\right| \\
\leq & \frac{1}{4}\left(M_{1}-m_{1}\right)\left(M_{2}-m_{2}\right) .
\end{aligned}
$$

We now state and prove our first theorem.

Theorem 3.1. Let $a, b, t \in \mathbb{T}, a<b$ and $f:[a, b] \rightarrow \mathbb{R}$ be differentiable. Suppose $f^{\Delta} \in C_{r d}$ and $\gamma \leq f^{\Delta}(t) \leq \Gamma$ for all $t \in[a, b]$. Then we have

$$
\begin{aligned}
& \mid f(x)-\frac{1}{\alpha+\beta}\left[\frac{\alpha}{x-a} \int_{a}^{x} f(\sigma(t)) \Delta t+\frac{\beta}{b-x} \int_{x}^{b} f(\sigma(t)) \Delta t\right] \\
& \quad-\frac{f(b)-f(a)}{(b-a)} \frac{1}{\alpha+\beta}\left[\frac{\alpha}{x-a} h_{2}(x, a)-\frac{\beta}{b-x} h_{2}(x, b)\right] \mid \\
& \leq \frac{1}{4}(b-a)(\Gamma-\gamma),
\end{aligned}
$$


for all $x \in[a, b]$.

Proof. Let $M_{1}=\sup _{a<t<b} P(x, t)$ and $m_{1}=\inf _{a<t<b} P(x, t)$. By the definition of $P(x, t)$ we have that $M_{1}=\frac{\alpha}{\alpha+\beta}$ and $m_{1}=\frac{-\beta}{\alpha+\beta}$. Thus, $M_{1}-m_{1}=1$ and

$$
m_{1} \leq P(x, t) \leq M_{1}
$$

Now by applying Lemma 3.2 to the functions $f(t):=P(x, t)$ and $g(t):=f^{\Delta}(t)$, we get

$$
\begin{aligned}
& \quad\left|\frac{1}{b-a} \int_{a}^{b} P(x, t) f^{\Delta}(t) \Delta t-\frac{1}{(b-a)^{2}} \int_{a}^{b} f^{\Delta}(t) \Delta t \int_{a}^{b} P(x, t) \Delta t\right| \\
& \leq \frac{1}{4}\left(M_{1}-m_{1}\right)(\Gamma-\gamma) .
\end{aligned}
$$

Thus,

$$
\begin{aligned}
& \left|\int_{a}^{b} P(x, t) f^{\Delta}(t) \Delta t-\frac{1}{b-a} \int_{a}^{b} f^{\Delta}(t) \Delta t \int_{a}^{b} P(x, t) \Delta t\right| \\
\leq & \frac{1}{4}(b-a)(\Gamma-\gamma) .
\end{aligned}
$$

By a simple computation, we have

$$
\int_{a}^{b} f^{\Delta}(t) \Delta t=f(b)-f(a)
$$

and

$$
\int_{a}^{b} P(x, t) \Delta t=\frac{1}{\alpha+\beta}\left[\frac{\alpha}{x-a} h_{2}(x, a)-\frac{\beta}{b-x} h_{2}(b, x)\right] .
$$

The desired inequality is obtained by substituting (3.1), (3.3) and (3.4) into (3.2).

Remark 3.1. We note that the inequality in Theorem 3.1 is not sharp. We will provide the sharp version in our next theorem.

Remark 3.2. If we set $\alpha=x-a$ and $\beta=b-x$ in Theorem 3.1, then we recapture Theorem 1.4.

If we apply Theorem 3.1 to the continuous, discrete and quantum calculus, we obtain some interesting inequalities which generalize the results in [20].

Corollary 3.1 (Continuous case). If we let $\mathbb{T}=\mathbb{R}$ in Theorem 3.1, then we have the inequality

$$
\begin{aligned}
& \mid f(x)-\frac{1}{\alpha+\beta}\left[\frac{\alpha}{x-a} \int_{a}^{x} f(t) d t+\frac{\beta}{b-x} \int_{x}^{b} f(t) d t\right] \\
& \quad-\frac{f(b)-f(a)}{2(b-a)} \frac{1}{\alpha+\beta}[\alpha(x-a)-\beta(b-x)] \mid \\
& \leq \frac{1}{4}(b-a)(\Gamma-\gamma),
\end{aligned}
$$


for all $x \in[a, b]$.

Corollary 3.2 (Discrete case). If we let $\mathbb{T}=\mathbb{Z}$ in Theorem 3.1, then we have the inequality

$$
\begin{aligned}
& \mid f(x)-\frac{1}{\alpha+\beta}\left[\frac{\alpha}{x-a} \sum_{t=a}^{x-1} f(t+1)+\frac{\beta}{b-x} \sum_{t=x}^{b-1} f(t+1)\right] \\
& \quad-\frac{f(b)-f(a)}{2(b-a)} \frac{1}{\alpha+\beta}[\alpha(x-a-1)-\beta(b-x+1)] \mid \\
& \leq \frac{1}{4}(b-a)(\Gamma-\gamma),
\end{aligned}
$$

for all $x \in\{a, a+1, \ldots, b-1, b\}$.

Corollary 3.3 (Quantum case). If we let $\mathbb{T}=q^{\mathbb{N}_{0}}, q>1$ in Theorem 3.1, then we have the inequality

$$
\begin{aligned}
& \quad \mid f(x)-\frac{1}{\alpha+\beta}\left[\frac{\alpha}{x-a} \int_{a}^{x} f(q t) d_{q} t+\frac{\beta}{b-x} \int_{x}^{b} f(q t) d_{q} t\right] \\
& \quad-\frac{f(b)-f(a)}{b-a} \frac{1}{(\alpha+\beta)(1+q)}[\alpha(x-q a)-\beta(q b-x)] \mid \\
& \leq
\end{aligned}
$$

for all $x \in[a, b]$.

In our next theorem, we provide a sharp bound for the inequality in Theorem 3.1. To do this, we need the following lemma which can be found in [21].

Lemma 3.3. ([21]) Let $a, b, x \in \mathbb{T}, f, g \in C_{r d}$ and $f, g:[a, b] \rightarrow \mathbb{R}$. Then if $\gamma \leq g(x) \leq \Gamma$ for all $x \in[a, b]$ and some $\gamma, \Gamma \in \mathbb{R}$, we have

$$
\begin{aligned}
& \left|\int_{a}^{b} f(t) g(t) \Delta t-\frac{1}{b-a} \int_{a}^{b} f(t) \Delta t \int_{a}^{b} g(t) \Delta t\right| \\
\leq & \frac{\Gamma-\gamma}{2} \int_{a}^{b}\left|f(t)-\frac{1}{b-a} \int_{a}^{b} f(s) \Delta s\right| \Delta t .
\end{aligned}
$$

Moreover, the inequality in (3.5) is sharp.

Theorem 3.2. Under the conditions of Lemma 3.1, we have the inequality

$$
\begin{aligned}
& \mid f(x)-\frac{1}{\alpha+\beta}\left[\frac{\alpha}{x-a} \int_{a}^{x} f(\sigma(t)) \Delta t+\frac{\beta}{b-x} \int_{x}^{b} f(\sigma(t)) \Delta t\right] \\
& \quad-\frac{f(b)-f(a)}{b-a} \frac{1}{\alpha+\beta}\left[\frac{\alpha}{x-a} h_{2}(x, a)-\frac{\beta}{b-x} h_{2}(x, b)\right] \mid \\
& \leq \frac{\Gamma-\gamma}{2} \int_{a}^{b}\left|P(x, t)-\frac{1}{b-a} \frac{1}{\alpha+\beta}\left[\frac{\alpha}{x-a} h_{2}(x, a)-\frac{\beta}{b-x} h_{2}(x, b)\right]\right| \Delta t,
\end{aligned}
$$


for all $x \in[a, b]$. Moreover, the inequality in (3.6) is sharp in the sense that the constant $\frac{1}{2}$ cannot be replaced by a smaller one.

Proof. By applying Lemma 3.3 to the functions $f(t):=P(x, t)$ and $g(t):=f^{\Delta}(t)$, we have

$$
\begin{aligned}
& \left|\int_{a}^{b} P(x, t) f^{\Delta}(t) \Delta t-\frac{1}{b-a} \int_{a}^{b} P(x, t) \Delta t \int_{a}^{b} f^{\Delta}(t) \Delta t\right| \\
\leq & \frac{\Gamma-\gamma}{2} \int_{a}^{b}\left|P(x, t)-\frac{1}{b-a} \int_{a}^{b} P(x, s) \Delta s\right| \Delta t .
\end{aligned}
$$

Now, we observe that

$$
\int_{a}^{b} f^{\Delta}(t) \Delta t=f(b)-f(a)
$$

and

$$
\int_{a}^{b} P(x, t) \Delta t=\frac{1}{\alpha+\beta}\left[\frac{\alpha}{x-a} h_{2}(x, a)-\frac{\beta}{b-x} h_{2}(b, x)\right] .
$$

The desired inequality is obtained by substituting (3.1), (3.8) and (3.9) in (3.7).

Remark 3.3. Let $\alpha=x-a$ and $\beta=b-x$ in Theorem 3.2. Then we recapture Theorem 1.5. Note that in this case $P(x, t)=\frac{K(x, t)}{b-a}$.

We now apply Theorem 3.2 to the continuous, discrete and quantum time scales to obtain some interesting inequalities which generalize the results in [21].

Corollary 3.4 (Continuous case). If we let $\mathbb{T}=\mathbb{R}$ in Theorem 3.2 , then we have the inequality

$$
\begin{aligned}
& \mid f(x)-\frac{1}{\alpha+\beta}\left[\frac{\alpha}{x-a} \int_{a}^{x} f(t) d t+\frac{\beta}{b-x} \int_{x}^{b} f(t) d t\right] \\
& \quad-\frac{f(b)-f(a)}{2(b-a)} \frac{1}{\alpha+\beta}[\alpha(x-a)-\beta(b-x)] \mid \\
& \leq \frac{\Gamma-\gamma}{2} \int_{a}^{b}\left|P(x, t)-\frac{1}{2(b-a)} \frac{1}{\alpha+\beta}[\alpha(x-a)-\beta(b-x)]\right| d t,
\end{aligned}
$$

for all $x \in[a, b]$. Moreover, the inequality in (3.10) is sharp in the sense that the constant $\frac{1}{2}$ cannot be replaced by a smaller one.

Corollary 3.5 (Discrete case). If we let $\mathbb{T}=\mathbb{Z}$ in Theorem 3.2 , then we have the inequality

$$
\begin{gathered}
\mid f(x)-\frac{1}{\alpha+\beta}\left[\frac{\alpha}{x-a} \sum_{t=a}^{x-1} f(t+1)+\frac{\beta}{b-x} \sum_{t=x}^{b-1} f(t+1)\right] \\
-\frac{f(b)-f(a)}{2(b-a)} \frac{1}{\alpha+\beta}[\alpha(x-a-1)-\beta(b-x+1)] \mid
\end{gathered}
$$




$$
\leq \frac{\Gamma-\gamma}{2} \sum_{t=a}^{b-1}\left|P(x, t)-\frac{1}{2(b-a)} \frac{1}{\alpha+\beta}[\alpha(x-a-1)-\beta(b-x+1)]\right|,
$$

for all $x \in\{a, a+1, \ldots, b-1, b\}$. Moreover, the inequality in (3.11) is sharp in the sense that the constant $\frac{1}{2}$ cannot be replaced by a smaller one.

Corollary 3.6 (Quantum case). If we let $\mathbb{T}=q^{\mathbb{N}_{0}}, q>1$, in Theorem 3.2, then we have the inequality

$$
\begin{aligned}
& \mid f(x)-\frac{1}{\alpha+\beta}\left[\frac{\alpha}{x-a} \int_{a}^{x} f(q t) d_{q} t+\frac{\beta}{b-x} \int_{x}^{b} f(q t) d_{q} t\right] \\
& \quad-\frac{f(b)-f(a)}{b-a} \frac{1}{(\alpha+\beta)(1+q)}[\alpha(x-q a)-\beta(q b-x)] \mid \\
& \leq \frac{\Gamma-\gamma}{2} \int_{a}^{b}\left|P(x, t)-\frac{1}{b-a} \frac{1}{(\alpha+\beta)(1+q)}[\alpha(x-q a)-\beta(q b-x)]\right| d_{q} t,
\end{aligned}
$$

for all $x \in[a, b]$. Moreover, the inequality in (3.12) is sharp in the sense that the constant $1 / 2$ cannot be replaced by a smaller one.

\section{A 2-Dimensional Ostrowski Inequality on Time Scales Involving a Combination of $\Delta$-Integral Means}

In what follows, we will let $\mathbb{T}_{1}$ and $\mathbb{T}_{2}$ denote two arbitrary time scales, with forward jump orperators $\sigma_{1}$ and $\sigma_{2}$ respectively. For an interval $[a, b],[a, b]_{\mathbb{T}_{i}}:=$ $[a, b] \cap \mathbb{T}_{i}, i=1,2$. For $a<b$ and $c<d$, we define the rectangle $[a, b]_{\mathbb{T}_{1}} \times[c, d]_{\mathbb{T}_{2}}$ as follows: $[a, b]_{\mathbb{T}_{1}} \times[c, d]_{\mathbb{T}_{2}}=\left\{(x, y): x \in[a, b]_{\mathbb{T}_{1}}, y \in[c, d]_{\mathbb{T}_{2}}\right\}$. For the sake of brevity, we will simply write $[a, b]$ instead of $[a, b]_{\mathbb{T}_{1}}$ and $[c, d]$ instead of $[c, d]_{\mathbb{T}_{2}}$. For more on the two-variable time scale calculus, we invite the interested reader to the papers $[6,7]$ and the references therein.

To prove our next theorem, we need the following lemma.

Lemma 4.1. Let $a, b, x, s \in \mathbb{T}_{1}, a<b, c, d, y, t \in \mathbb{T}_{2}, c<d$ and let $f:[a, b] \times[c, d] \rightarrow$ $\mathbb{R}$ be such that the partial derivatives $\frac{\partial f(s, t)}{\Delta_{1} s}, \frac{\partial f(s, t)}{\Delta_{2} t}, \frac{\partial^{2} f(s, t)}{\Delta_{2} t \Delta_{1} s}$ exist and are continuous on $[a, b] \times[c, d]$. Then we have

$$
\begin{aligned}
& f(x, y)-\frac{1}{\left(\alpha_{1}+\beta_{1}\right)\left(\alpha_{2}+\beta_{2}\right)}\left[\frac{\alpha_{2} \alpha_{1}}{(y-c)(x-a)} \int_{a}^{x} \int_{c}^{y} f\left(\sigma_{1}(s), \sigma_{2}(t)\right) \Delta_{2} t \Delta_{1} s\right. \\
& +\frac{\beta_{2} \alpha_{1}}{(d-y)(x-a)} \int_{a}^{x} \int_{y}^{d} f\left(\sigma_{1}(s), \sigma_{2}(t)\right) \Delta_{2} t \Delta_{1} s \\
& +\frac{\alpha_{2} \beta_{1}}{(y-c)(b-x)} \int_{x}^{b} \int_{c}^{y} f\left(\sigma_{1}(s), \sigma_{2}(t)\right) \Delta_{2} t \Delta_{1} s \\
& \left.+\frac{\beta_{2} \beta_{1}}{(d-y)(b-x)} \int_{x}^{b} \int_{y}^{d} f\left(\sigma_{1}(s), \sigma_{2}(t)\right) \Delta_{2} t \Delta_{1} s\right]
\end{aligned}
$$




$$
\begin{aligned}
= & \frac{\alpha_{1}}{\left(\alpha_{1}+\beta_{1}\right)(x-a)} \int_{a}^{x} \int_{c}^{d} P_{2}(y, t) \frac{\partial f\left(\sigma_{1}(s), t\right)}{\Delta_{2} t} \Delta_{2} t \Delta_{1} s \\
& +\frac{\beta_{1}}{\left(\alpha_{1}+\beta_{1}\right)(b-x)} \int_{x}^{b} \int_{c}^{d} P_{2}(y, t) \frac{\partial f\left(\sigma_{1}(s), t\right)}{\Delta_{2} t} \Delta_{2} t \Delta_{1} s \\
& +\frac{\alpha_{2}}{\left(\alpha_{2}+\beta_{2}\right)(y-c)} \int_{c}^{y} \int_{a}^{b} P_{1}(x, s) \frac{\partial f\left(s, \sigma_{2}(t)\right)}{\Delta_{1} s} \Delta_{1} s \Delta_{2} t \\
& +\frac{\beta_{2}}{\left(\alpha_{2}+\beta_{2}\right)(d-y)} \int_{y}^{d} \int_{a}^{b} P_{1}(x, s) \frac{\partial f\left(s, \sigma_{2}(t)\right)}{\Delta_{1} s} \Delta_{1} s \Delta_{2} t \\
& +\int_{c}^{d} \int_{a}^{b} P_{1}(x, s) P_{2}(y, t) \frac{\partial^{2} f(s, t)}{\Delta_{2} t \Delta_{1} s} \Delta_{1} s \Delta_{2} t,
\end{aligned}
$$

for all $x \in[a, b]$ and $y \in[c, d]$, where

$$
\begin{gathered}
P_{1}(x, s)= \begin{cases}\frac{\alpha_{1}}{\alpha_{1}+\beta_{1}}\left(\frac{s-a}{x-a}\right), & s \in[a, x), \\
\frac{-\beta_{1}}{\alpha_{1}+\beta_{1}}\left(\frac{b-s}{b-x}\right), & s \in[x, b],\end{cases} \\
P_{2}(y, t)= \begin{cases}\frac{\alpha_{2}}{\alpha_{2}+\beta_{2}}\left(\frac{t-c}{y-c}\right), & t \in[c, y), \\
\frac{-\beta_{2}}{\alpha_{2}+\beta_{2}}\left(\frac{d-t}{d-y}\right), & t \in[y, d],\end{cases}
\end{gathered}
$$

$\alpha_{1}, \beta_{1}, \alpha_{2}$ and $\beta_{2}$ are nonnegative numbers with $\alpha_{1}+\beta_{1}>0$ and $\alpha_{2}+\beta_{2}>0$.

Proof. By applying Lemma 3.1 to the partial map $f(\cdot, y), y \in[c, d]$, we have for $x \in[a, b]$

$$
\begin{aligned}
f(x, y)= & \frac{1}{\alpha_{1}+\beta_{1}}\left[\frac{\alpha_{1}}{x-a} \int_{a}^{x} f\left(\sigma_{1}(s), y\right) \Delta_{1} s+\frac{\beta_{1}}{b-x} \int_{x}^{b} f\left(\sigma_{1}(s), y\right) \Delta_{1} s\right] \\
& +\int_{a}^{b} P_{1}(x, s) \frac{\partial f(s, y)}{\Delta_{1} s} \Delta_{1} s .
\end{aligned}
$$

If we apply Lemma 3.1 to the maps $f\left(\sigma_{1}(s), \cdot\right)$ and $\frac{\partial f(s, \cdot)}{\Delta_{1} s}$, we have

$$
\begin{aligned}
f\left(\sigma_{1}(s), y\right)= & \frac{1}{\alpha_{2}+\beta_{2}}\left[\frac{\alpha_{2}}{y-c} \int_{c}^{y} f\left(\sigma_{1}(s), \sigma_{2}(t)\right) \Delta_{2} t+\frac{\beta_{2}}{d-y} \int_{y}^{d} f\left(\sigma_{1}(s), \sigma_{2}(t)\right) \Delta_{2} t\right] \\
& +\int_{c}^{d} P_{2}(y, t) \frac{\partial f\left(\sigma_{1}(s), t\right)}{\Delta_{2} t} \Delta_{2} t
\end{aligned}
$$

and

$$
\begin{aligned}
\frac{\partial f(s, y)}{\Delta_{1} s}= & \frac{1}{\alpha_{2}+\beta_{2}}\left[\frac{\alpha_{2}}{y-c} \int_{c}^{y} \frac{\partial f\left(s, \sigma_{2}(t)\right)}{\Delta_{1} s} \Delta_{2} t+\frac{\beta_{2}}{d-y} \int_{y}^{d} \frac{\partial f\left(s, \sigma_{2}(t)\right)}{\Delta_{1} s} \Delta_{2} t\right] \\
& +\int_{c}^{d} P_{2}(y, t) \frac{\partial^{2} f(s, t)}{\Delta_{2} t \Delta_{1} s} \Delta_{2} t
\end{aligned}
$$


By substituting (4.3) and (4.4) into (4.2) we obtain,

$$
\begin{aligned}
f(x, y)= & \frac{1}{\alpha_{1}+\beta_{1}}\left[\frac { \alpha _ { 1 } } { x - a } \int _ { a } ^ { x } \left(\frac { 1 } { \alpha _ { 2 } + \beta _ { 2 } } \left[\frac { \alpha _ { 2 } } { y - c } \int _ { c } ^ { y } f \left(\sigma_{1}(s), \sigma_{2}(t) \Delta_{2} t\right.\right.\right.\right. \\
& \left.\left.+\frac{\beta_{2}}{d-y} \int_{y}^{d} f\left(\sigma_{1}(s), \sigma_{2}(t)\right) \Delta_{2} t\right]+\int_{c}^{d} P_{2}(y, t) \frac{\partial f\left(\sigma_{1}(s), t\right)}{\Delta_{2} t} \Delta_{2} t\right) \Delta_{1} s \\
& +\frac{\beta_{1}}{b-x} \int_{x}^{b}\left(\frac { 1 } { \alpha _ { 2 } + \beta _ { 2 } } \left[\frac{\alpha_{2}}{y-c} \int_{c}^{y} f\left(\sigma_{1}(s), \sigma_{2}(t)\right) \Delta_{2} t\right.\right. \\
& \left.\left.\left.+\frac{\beta_{2}}{d-y} \int_{y}^{d} f\left(\sigma_{1}(s), \sigma_{2}(t)\right) \Delta_{2} t\right]+\int_{c}^{d} P_{2}(y, t) \frac{\partial f\left(\sigma_{1}(s), t\right)}{\Delta_{2} t} \Delta_{2} t\right) \Delta_{1} s\right] \\
& +\int_{a}^{b} P_{1}(x, s)\left(\frac { 1 } { \alpha _ { 2 } + \beta _ { 2 } } \left[\frac{\alpha_{2}}{y-c} \int_{c}^{y} \frac{\partial f\left(s, \sigma_{2}(t)\right)}{\Delta_{1} s} \Delta_{2} t\right.\right. \\
& \left.\left.+\frac{\beta_{2}}{d-y} \int_{y}^{d} \frac{\partial f\left(s, \sigma_{2}(t)\right)}{\Delta_{1} s} \Delta_{2} t\right]+\int_{c}^{d} P_{2}(y, t) \frac{\partial^{2} f(s, t)}{\Delta_{2} t \Delta_{1} s} \Delta_{2} t\right) \Delta_{1} s .
\end{aligned}
$$

By rearranging the terms we get

$$
\begin{aligned}
f(x, y)= & \frac{1}{\left(\alpha_{1}+\beta_{1}\right)\left(\alpha_{2}+\beta_{2}\right)}\left[\frac{\alpha_{2} \alpha_{1}}{(y-c)(x-a)} \int_{a}^{x} \int_{c}^{y} f\left(\sigma_{1}(s), \sigma_{2}(t)\right) \Delta_{2} t \Delta_{1} s\right. \\
& +\frac{\beta_{2} \alpha_{1}}{(d-y)(x-a)} \int_{a}^{x} \int_{y}^{d} f\left(\sigma_{1}(s), \sigma_{2}(t)\right) \Delta_{2} t \Delta_{1} s \\
& +\frac{\alpha_{2} \beta_{1}}{(y-c)(b-x)} \int_{x}^{b} \int_{c}^{y} f\left(\sigma_{1}(s), \sigma_{2}(t)\right) \Delta_{2} t \Delta_{1} s \\
& \left.+\frac{\beta_{2} \beta_{1}}{(d-y)(b-x)} \int_{x}^{b} \int_{y}^{d} f\left(\sigma_{1}(s), \sigma_{2}(t)\right) \Delta_{2} t \Delta_{1} s\right] \\
& +\frac{\alpha_{1}}{\left(\alpha_{1}+\beta_{1}\right)(x-a)} \int_{a}^{x} \int_{c}^{d} P_{2}(y, t) \frac{\partial f\left(\sigma_{1}(s), t\right)}{\Delta_{2} t} \Delta_{2} t \Delta_{1} s \\
& +\frac{\beta_{1}}{\left(\alpha_{1}+\beta_{1}\right)(b-x)} \int_{x}^{b} \int_{c}^{d} P_{2}(y, t) \frac{\partial f\left(\sigma_{1}(s), t\right)}{\Delta_{2} t} \Delta_{2} t \Delta_{1} s \\
& +\frac{\alpha_{2}}{\left(\alpha_{2}+\beta_{2}\right)(y-c)} \int_{c}^{y} \int_{a}^{b} P_{1}(x, s) \frac{\partial f\left(s, \sigma_{2}(t)\right)}{\Delta_{1} s} \Delta_{1} s \Delta_{2} t \\
& +\frac{\beta_{2}}{\left(\alpha_{2}+\beta_{2}\right)(d-y)} \int_{y}^{d} \int_{a}^{b} P_{1}(x, s) \frac{\partial f\left(s, \sigma_{2}(t)\right)}{\Delta_{1} s} \Delta_{1} s \Delta_{2} t \\
& +\int_{c}^{d} \int_{a}^{b} P_{1}(x, s) P_{2}(y, t) \frac{\partial^{2} f(s, t)}{\Delta_{2} t \Delta_{1} s} \Delta_{1} s \Delta_{2} t .
\end{aligned}
$$

The identity in (4.1) follows directly from (4.5). 
Theorem 4.1. Under the conditions of Lemma 4.1, we have the inequality

$$
\begin{aligned}
& \mid f(x, y)-\frac{1}{\left(\alpha_{1}+\beta_{1}\right)\left(\alpha_{2}+\beta_{2}\right)}\left[\frac{\alpha_{2} \alpha_{1}}{(y-c)(x-a)} \int_{a}^{x} \int_{c}^{y} f\left(\sigma_{1}(s), \sigma_{2}(t)\right) \Delta_{2} t \Delta_{1} s\right. \\
& +\frac{\beta_{2} \alpha_{1}}{(d-y)(x-a)} \int_{a}^{x} \int_{y}^{d} f\left(\sigma_{1}(s), \sigma_{2}(t)\right) \Delta_{2} t \Delta_{1} s \\
& +\frac{\alpha_{2} \beta_{1}}{(y-c)(b-x)} \int_{x}^{b} \int_{c}^{y} f\left(\sigma_{1}(s), \sigma_{2}(t)\right) \Delta_{2} t \Delta_{1} s \\
& \left.+\frac{\beta_{2} \beta_{1}}{(d-y)(b-x)} \int_{x}^{b} \int_{y}^{d} f\left(\sigma_{1}(s), \sigma_{2}(t)\right) \Delta_{2} t \Delta_{1} s\right] \mid \\
& \leq \\
& \quad \frac{M_{2}}{\alpha_{2}+\beta_{2}}\left[\frac{\alpha_{2}}{y-c} h_{2}(y, c)+\frac{\beta_{2}}{d-y} h_{2}(y, d)\right] \\
& +\frac{M_{1}}{\alpha_{1}+\beta_{1}}\left[\frac{\alpha_{1}}{x-a} h_{2}(x, a)+\frac{\beta_{1}}{b-x} h_{2}(x, b)\right] \\
& +\frac{M_{3}}{\left(\alpha_{1}+\beta_{1}\right)\left(\alpha_{2}+\beta_{2}\right)}\left[\frac{\alpha_{1}}{x-a} h_{2}(x, a)+\frac{\beta_{1}}{b-x} h_{2}(x, b)\right] \\
& \quad \times\left[\frac{\alpha_{2}}{y-c} h_{2}(y, c)+\frac{\beta_{2}}{d-y} h_{2}(y, d)\right],
\end{aligned}
$$

for all $x \in[a, b]$ and $y \in[c, d]$, where

$$
\begin{gathered}
M_{1}=\sup _{a<s<b}\left|\frac{\partial f(s, t)}{\Delta_{1} s}\right|<\infty, \quad M_{2}=\sup _{c<t<d}\left|\frac{\partial f(s, t)}{\Delta_{2} t}\right|<\infty \quad \text { and } \\
M_{3}=\sup _{a<s<b, c<t<d}\left|\frac{\partial^{2} f(s, t)}{\Delta_{2} t \Delta_{1} s}\right|<\infty .
\end{gathered}
$$

Proof. From Lemma 4.1, we have that

$$
\begin{aligned}
& f(x, y)-\frac{1}{\left(\alpha_{1}+\beta_{1}\right)\left(\alpha_{2}+\beta_{2}\right)}\left[\frac{\alpha_{2} \alpha_{1}}{(y-c)(x-a)} \int_{a}^{x} \int_{c}^{y} f\left(\sigma_{1}(s), \sigma_{2}(t)\right) \Delta_{2} t \Delta_{1} s\right. \\
& +\frac{\beta_{2} \alpha_{1}}{(d-y)(x-a)} \int_{a}^{x} \int_{y}^{d} f\left(\sigma_{1}(s), \sigma_{2}(t)\right) \Delta_{2} t \Delta_{1} s \\
& +\frac{\alpha_{2} \beta_{1}}{(y-c)(b-x)} \int_{x}^{b} \int_{c}^{y} f\left(\sigma_{1}(s), \sigma_{2}(t)\right) \Delta_{2} t \Delta_{1} s \\
& \left.+\frac{\beta_{2} \beta_{1}}{(d-y)(b-x)} \int_{x}^{b} \int_{y}^{d} f\left(\sigma_{1}(s), \sigma_{2}(t)\right) \Delta_{2} t \Delta_{1} s\right] \\
& =\frac{\alpha_{1}}{\left(\alpha_{1}+\beta_{1}\right)(x-a)} \int_{a}^{x} \int_{c}^{d} P_{2}(y, t) \frac{\partial f\left(\sigma_{1}(s), t\right)}{\Delta_{2} t} \Delta_{2} t \Delta_{1} s \\
& +\frac{\beta_{1}}{\left(\alpha_{1}+\beta_{1}\right)(b-x)} \int_{x}^{b} \int_{c}^{d} P_{2}(y, t) \frac{\partial f\left(\sigma_{1}(s), t\right)}{\Delta_{2} t} \Delta_{2} t \Delta_{1} s
\end{aligned}
$$




$$
\begin{aligned}
& +\frac{\alpha_{2}}{\left(\alpha_{2}+\beta_{2}\right)(y-c)} \int_{c}^{y} \int_{a}^{b} P_{1}(x, s) \frac{\partial f\left(s, \sigma_{2}(t)\right)}{\Delta_{1} s} \Delta_{1} s \Delta_{2} t \\
& +\frac{\beta_{2}}{\left(\alpha_{2}+\beta_{2}\right)(d-y)} \int_{y}^{d} \int_{a}^{b} P_{1}(x, s) \frac{\partial f\left(s, \sigma_{2}(t)\right)}{\Delta_{1} s} \Delta_{1} s \Delta_{2} t \\
& +\int_{c}^{d} \int_{a}^{b} P_{1}(x, s) P_{2}(y, t) \frac{\partial^{2} f(s, t)}{\Delta_{2} t \Delta_{1} s} \Delta_{1} s \Delta_{2} t .
\end{aligned}
$$

By taking the absolute values on both sides of (4.6) and applying item (v) of Theorem 2.2 , we obtain

$$
\begin{aligned}
\mid f(x, y)-\frac{1}{\left(\alpha_{1}+\beta_{1}\right)\left(\alpha_{2}+\beta_{2}\right)}\left[\frac{\alpha_{2} \alpha_{1}}{(y-c)(x-a)} \int_{a}^{x} \int_{c}^{y} f\left(\sigma_{1}(s), \sigma_{2}(t)\right) \Delta_{2} t \Delta_{1} s\right. \\
+\frac{\beta_{2} \alpha_{1}}{(d-y)(x-a)} \int_{a}^{x} \int_{y}^{d} f\left(\sigma_{1}(s), \sigma_{2}(t)\right) \Delta_{2} t \Delta_{1} s \\
+\frac{\alpha_{2} \beta_{1}}{(y-c)(b-x)} \int_{x}^{b} \int_{c}^{y} f\left(\sigma_{1}(s), \sigma_{2}(t)\right) \Delta_{2} t \Delta_{1} s \\
\left.+\frac{\beta_{2} \beta_{1}}{(d-y)(b-x)} \int_{x}^{b} \int_{y}^{d} f\left(\sigma_{1}(s), \sigma_{2}(t)\right) \Delta_{2} t \Delta_{1} s\right] \mid \\
\leq \\
+\frac{\alpha_{1} M_{2}}{\left(\alpha_{1}+\beta_{1}\right)(x-a)} \int_{a}^{x} \int_{c}^{d}\left|P_{2}(y, t)\right| \Delta_{2} t \Delta_{1} s \\
+\frac{\beta_{1} M_{2}}{\left(\alpha_{1}+\beta_{1}\right)(b-x)} \int_{x}^{b} \int_{c}^{d}\left|P_{2}(y, t)\right| \Delta_{2} t \Delta_{1} s \\
+\frac{\alpha_{2} M_{1}}{\left(\alpha_{2}+\beta_{2}\right)(y-c)} \int_{c}^{y} \int_{a}^{b}\left|P_{1}(x, s)\right| \Delta_{1} s \Delta_{2} t \\
+\frac{\beta_{2} M_{1}}{\left(\alpha_{2}+\beta_{2}\right)(d-y)} \int_{y}^{d} \int_{a}^{b}\left|P_{1}(x, s)\right| \Delta_{1} s \Delta_{2} t \\
+M_{3} \int_{c}^{d} \int_{a}^{b}\left|P_{1}(x, s)\right|\left|P_{2}(y, t)\right| \Delta_{1} s \Delta_{2} t \\
=M_{2} \int_{c}^{d}\left|P_{2}(y, t)\right| \Delta_{2} t+M_{1} \int_{a}^{b}\left|P_{1}(x, s)\right| \Delta_{1} s \\
+M_{3} \int_{c}^{d} \int_{a}^{b}\left|P_{1}(x, s)\right|\left|P_{2}(y, t)\right| \Delta_{1} s \Delta_{2} t .
\end{aligned}
$$

That is,

$$
\begin{aligned}
& \mid f(x, y)-\frac{1}{\left(\alpha_{1}+\beta_{1}\right)\left(\alpha_{2}+\beta_{2}\right)}\left[\frac{\alpha_{2} \alpha_{1}}{(y-c)(x-a)} \int_{a}^{x} \int_{c}^{y} f\left(\sigma_{1}(s), \sigma_{2}(t)\right) \Delta_{2} t \Delta_{1} s\right. \\
& +\frac{\beta_{2} \alpha_{1}}{(d-y)(x-a)} \int_{a}^{x} \int_{y}^{d} f\left(\sigma_{1}(s), \sigma_{2}(t)\right) \Delta_{2} t \Delta_{1} s
\end{aligned}
$$




$$
\begin{aligned}
& +\frac{\alpha_{2} \beta_{1}}{(y-c)(b-x)} \int_{x}^{b} \int_{c}^{y} f\left(\sigma_{1}(s), \sigma_{2}(t)\right) \Delta_{2} t \Delta_{1} s \\
& \left.+\frac{\beta_{2} \beta_{1}}{(d-y)(b-x)} \int_{x}^{b} \int_{y}^{d} f\left(\sigma_{1}(s), \sigma_{2}(t)\right) \Delta_{2} t \Delta_{1} s\right] \mid \\
\leq & M_{1} \int_{a}^{b}\left|P_{1}(x, s)\right| \Delta_{1} s+M_{2} \int_{c}^{d}\left|P_{2}(y, t)\right| \Delta_{2} t+M_{3} \int_{c}^{d} \int_{a}^{b}\left|P_{1}(x, s)\right|\left|P_{2}(y, t)\right| \Delta_{1} s \Delta_{2} t .
\end{aligned}
$$

The desired inequality follows from (4.7) by using the fact that

$$
\int_{a}^{b}\left|P_{1}(x, s)\right| \Delta_{1} s=\frac{1}{\alpha_{1}+\beta_{1}}\left[\frac{\alpha_{1}}{x-a} h_{2}(x, a)+\frac{\beta_{1}}{b-x} h_{2}(x, b)\right]
$$

and

$$
\int_{c}^{d}\left|P_{2}(y, t)\right| \Delta_{2} t=\frac{1}{\alpha_{2}+\beta_{2}}\left[\frac{\alpha_{2}}{y-c} h_{2}(y, c)+\frac{\beta_{2}}{d-y} h_{2}(y, d)\right] .
$$

The following corollary is Theorem 4 in [17] but we state it here for completion.

Corollary 4.1 ([17]). Let $a, b, x, s \in \mathbb{T}_{1}, a<b, c, d, y, t \in \mathbb{T}_{2}, c<d$ and let $f$ : $[a, b] \times[c, d] \rightarrow \mathbb{R}$ be such that the partial derivatives $\frac{\partial f(s, t)}{\Delta_{1} s}, \frac{\partial f(s, t)}{\Delta_{2} t}, \frac{\partial^{2} f(s, t)}{\Delta_{2} t \Delta_{1} s}$ exist and are continuous on $[a, b] \times[c, d]$. Then we have the inequality

$$
\begin{aligned}
& \left|f(x, y)-\frac{1}{(b-a)(d-c)} \int_{a}^{b} \int_{c}^{d} f\left(\sigma_{1}(s), \sigma_{2}(t)\right) \Delta_{2} t \Delta_{1} s\right| \\
& \leq \frac{M_{2}}{d-c}\left[h_{2}(y, c)+h_{2}(y, d)\right]+\frac{M_{1}}{b-a}\left[h_{2}(x, a)+h_{2}(x, b)\right] \\
& \quad+\frac{M_{3}}{(b-a)(d-c)}\left[h_{2}(x, a)+h_{2}(x, b)\right]\left[h_{2}(y, c)+h_{2}(y, d)\right],
\end{aligned}
$$

for all $x \in[a, b]$ and $y \in[c, d]$, where

$$
\begin{gathered}
M_{1}=\sup _{a<s<b}\left|\frac{\partial f(s, t)}{\Delta_{1} s}\right|<\infty, \quad M_{2}=\sup _{c<t<d}\left|\frac{\partial f(s, t)}{\Delta_{2} t}\right|<\infty \quad \text { and } \\
M_{3}=\sup _{a<s<b, c<t<d}\left|\frac{\partial^{2} f(s, t)}{\Delta_{2} t \Delta_{1} s}\right|<\infty .
\end{gathered}
$$

Proof. Let $\alpha_{1}=x-a, \beta_{1}=b-x, \alpha_{2}=y-c$ and $\beta_{2}=d-y$ in Theorem 4.1.

Now, we apply Theorem 4.1 to the continuous, discrete and quantum time scales to obtain some interesting inequalities which generalize the results in [17].

Corollary 4.2 (Continuous case). If we let $\mathbb{T}_{1}=\mathbb{T}_{2}=\mathbb{R}$ in Theorem 4.1 , then we have

$$
\begin{aligned}
& \mid f(x, y)-\frac{1}{\left(\alpha_{1}+\beta_{1}\right)\left(\alpha_{2}+\beta_{2}\right)}\left[\frac{\alpha_{2} \alpha_{1}}{(y-c)(x-a)} \int_{a}^{x} \int_{c}^{y} f(s, t) d t d s\right. \\
& +\frac{\beta_{2} \alpha_{1}}{(d-y)(x-a)} \int_{a}^{x} \int_{y}^{d} f(s, t) d t d s+\frac{\alpha_{2} \beta_{1}}{(y-c)(b-x)} \int_{x}^{b} \int_{c}^{y} f(s, t) d t d s
\end{aligned}
$$




$$
\begin{aligned}
& \left.+\frac{\beta_{2} \beta_{1}}{(d-y)(b-x)} \int_{x}^{b} \int_{y}^{d} f(s, t) d t d s\right] \mid \\
\leq & \frac{M_{2}}{2\left(\alpha_{2}+\beta_{2}\right)}\left[\alpha_{2}(y-c)+\beta_{2}(d-y)\right]+\frac{M_{1}}{2\left(\alpha_{1}+\beta_{1}\right)}\left[\alpha_{1}(x-a)+\beta_{1}(b-x)\right] \\
& +\frac{M_{3}}{4\left(\alpha_{1}+\beta_{1}\right)\left(\alpha_{2}+\beta_{2}\right)}\left[\alpha_{1}(x-a)+\beta_{1}(b-x)\right]\left[\alpha_{2}(y-c)+\beta_{2}(d-y)\right],
\end{aligned}
$$

for all $x \in[a, b]$ and $y \in[c, d]$, where

$$
\begin{gathered}
M_{1}=\sup _{a<s<b}\left|\frac{\partial f(s, t)}{\partial s}\right|<\infty, \quad M_{2}=\sup _{c<t<d}\left|\frac{\partial f(s, t)}{\partial t}\right|<\infty \quad \text { and } \\
M_{3}=\sup _{a<s<b, c<t<d}\left|\frac{\partial^{2} f(s, t)}{\partial t \partial s}\right|<\infty .
\end{gathered}
$$

Corollary 4.3 (Discrete case). If we let $\mathbb{T}_{1}=\mathbb{T}_{2}=\mathbb{Z}$ in Theorem 4.1 , then we have

$$
\begin{aligned}
& \mid f(x, y)-\frac{1}{\left(\alpha_{1}+\beta_{1}\right)\left(\alpha_{2}+\beta_{2}\right)}\left[\frac{\alpha_{2} \alpha_{1}}{(y-c)(x-a)} \sum_{s=a}^{x-1} \sum_{t=c}^{y-1} f(s+1, t+1)\right. \\
& \quad+\frac{\beta_{2} \alpha_{1}}{(d-y)(x-a)} \sum_{s=a}^{x-1} \sum_{t=y}^{d-1} f(s+1, t+1)+\frac{\alpha_{2} \beta_{1}}{(y-c)(b-x)} \sum_{s=x}^{b-1} \sum_{t=c}^{y-1} f(s+1, t+1) \\
& \left.\quad+\frac{\beta_{2} \beta_{1}}{(d-y)(b-x)} \sum_{s=x}^{b-1} \sum_{t=y}^{d-1} f(s+1, t+1)\right] \mid \\
& \leq \\
& \quad \frac{M_{2}}{2\left(\alpha_{2}+\beta_{2}\right)}\left[\alpha_{2}(y-c-1)+\beta_{2}(d-y+1)\right] \\
& \quad+\frac{M_{1}}{2\left(\alpha_{1}+\beta_{1}\right)}\left[\alpha_{1}(x-a-1)+\beta_{1}(b-x+1)\right] \\
& \quad+\frac{M_{3}}{4\left(\alpha_{1}+\beta_{1}\right)\left(\alpha_{2}+\beta_{2}\right)}\left[\alpha_{1}(x-a-1)+\beta_{1}(b-x+1)\right] \\
& \quad \times\left[\alpha_{2}(y-c-1)+\beta_{2}(d-y+1)\right],
\end{aligned}
$$

for all $x \in\{a, a+1, \ldots, b-1, b\}$ and $y \in\{c, c+1, \ldots, d-1, d\}$, where

$$
M_{1}=\sup _{a<s<b}|f(s+1, t)-f(s, t)|<\infty, \quad M_{2}=\sup _{c<t<d}|f(s, t+1)-f(s, t)|<\infty
$$

and

$$
M_{3}=\sup _{a<s<b, c<t<d}|f(s+1, t+1)-f(s+1, t)-f(s, t+1)+f(s, t)|<\infty .
$$

Corollary 4.4 (Quantum case). If we let $\mathbb{T}_{1}=q_{1}^{\mathbb{N}_{0}}, q_{1}>1$ and $\mathbb{T}_{2}=q_{2}^{\mathbb{N}_{0}}, q_{2}>1$ in Theorem 4.1, then we have

$$
\mid f(x, y)-\frac{1}{\left(\alpha_{1}+\beta_{1}\right)\left(\alpha_{2}+\beta_{2}\right)}\left[\frac{\alpha_{2} \alpha_{1}}{(y-c)(x-a)} \int_{a}^{x} \int_{c}^{y} f\left(q_{1} s, q_{2} t\right) d_{q_{2}} t d_{q_{1}} s\right.
$$




$$
\begin{aligned}
& +\frac{\beta_{2} \alpha_{1}}{(d-y)(x-a)} \int_{a}^{x} \int_{y}^{d} f\left(q_{1} s, q_{2} t\right) d_{q_{2}} t d_{q_{1}} s \\
& +\frac{\alpha_{2} \beta_{1}}{(y-c)(b-x)} \int_{x}^{b} \int_{c}^{y} f\left(q_{1} s, q_{2} t\right) d_{q_{2}} t d_{q_{1}} s \\
& \left.+\frac{\beta_{2} \beta_{1}}{(d-y)(b-x)} \int_{x}^{b} \int_{y}^{d} f\left(q_{1} s, q_{2} t\right) d_{q_{2}} t d_{q_{1}} s\right] \mid \\
& \leq \frac{M_{2}}{\alpha_{2}+\beta_{2}}\left[\frac{\alpha_{2}\left(y-q_{2} c\right)+\beta_{2}\left(q_{2} d-y\right)}{1+q_{2}}\right]+\frac{M_{1}}{\alpha_{1}+\beta_{1}}\left[\frac{\alpha_{1}\left(x-q_{1} a\right)+\beta_{1}\left(q_{1} b-x\right)}{1+q_{2}}\right] \\
& +\frac{M_{3}}{\left(\alpha_{1}+\beta_{1}\right)\left(\alpha_{2}+\beta_{2}\right)}\left[\frac{\alpha_{1}\left(x-q_{1} a\right)+\beta_{1}\left(q_{1} b-x\right)}{1+q_{2}}\right]\left[\frac{\alpha_{2}\left(y-q_{2} c\right)+\beta_{2}\left(q_{2} d-y\right)}{1+q_{2}}\right],
\end{aligned}
$$

for all $x \in[a, b]$ and $y \in[c, d]$ with

$$
\begin{aligned}
M_{1} & =\sup _{a<s<b}\left|\frac{f\left(q_{1} s, t\right)-f(s, t)}{\left(q_{1}-1\right) s}\right|<\infty, \quad M_{2}=\sup _{c<t<d}\left|\frac{f\left(s, q_{2} t\right)-f(s, t)}{\left(q_{2}-1\right) t}\right|<\infty \\
\text { and } M_{3} & =\sup _{a<s<b, c<t<d}\left|\frac{f\left(q_{1} s, q_{2} t\right)-f\left(q_{1} s, t\right)-f\left(s, q_{2} t\right)+f(s, t)}{\left(q_{1}-1\right)\left(q_{2}-1\right) s t}\right|<\infty .
\end{aligned}
$$

\section{Conclusion}

In this work, we established some new Ostrowski-Grüss and 2D Ostrowski type inequalities on time scales involving a combination of $\Delta$-integral means. In addition, we apply our results to the continuous, discrete and quantum calculus to obtain some novel inequalites in this direction.

\section{REFERENCES}

[1] R. Agarwal, M. Bohner and A. Peterson, Inequalities on time scales: a survey, Math. Inequal. Appl. 4(4) (2001), 535-557.

[2] M. Bohner and T. Matthews, The Grüss inequality on time scales, Commun. Math. Anal. 3(1) (2007), 1-8.

[3] M. Bohner and T. Matthews, Ostrowski inequalities on time scales, Journal of Inequalities in Pure and Applied Mathematics 9(1) 2008, Article ID 6.

[4] M. Bohner and A. Peterson, Dynamic Equations on Time Scales, Birkhäuser Boston, Boston, MA, 2001.

[5] M. Bohner and A. Peterson, Advances in Dynamic Equations on Time Series, Birkhäuser Boston, Boston, MA, 2003.

[6] M. Bohner and G. S. Guseinov, Partial differentiation on time scales, Dynam. Systems Appl. 13(3-4) (2004), 351-379.

[7] M. Bohner and G. S. Guseinov, Multiple integration on time scales, Dynam. Systems Appl. 14(3-4) (2005), 579-606.

[8] P. Cerone, A new Ostrowski type inequality involving integral means over end intervals, Tamkang J. Math. 33 (2002), 109-118.

[9] S. S. Dragomir and S. Wang, An inequality of Ostrowski-Grüss type and its applications to the estimation of error bounds for some special means and for some numerical quadrature rules, Comput. Math. Appl. 33 (1997), 15-20. 
[10] S. S. Dragomir and N. S. Barnett, An Ostrowski type inequality for mappings whose second derivatives are bounded and applications, Indian J. Math. 66(1-4) (1999), 237-245.

[11] S. Hilger, Ein Masskettenkalkül mit Anwendung auf Zentrumsmannigfaltigkeiten, Ph.D. Thesis, Universität Würzburg, Würzburg, Germany, 1988.

[12] S. Hussain, M. A. Latif and M. Alomari, Generalized double integral Ostrowski type inequalities on time scales, Appl. Math. Lett. 24(8) (2011), 1461-1467.

[13] W. Irshad, M. I. Bhatti and M. Muddassar, Some Ostrowski type integral inequalities for double integral on time scales, J. Comput. Anal. Appl. 20(5) (2016), 914-927.

[14] Y. Jiang, H. Rüzgar, W. J. Liu and A. Tuna, Some new generalizations of Ostrowski type inequalities on time scales involving combination of $\Delta$-integral means, J. Nonlinear Sci. Appl. 7 (2014), 311-324.

[15] S. Kermausuor, E. R. Nwaeze and D. F. M. Torres, Generalized weighted Ostrowski and Ostrowski-Grüss type inequalities on time scale via a parameter function, J. Math. Inequal. 11 (2017), 1185-1199.

[16] V. Lakshmikantham, S. Sivasundaram and B. Kaymakcalan, Dynamic Systems on Measure Chains, Mathematics and its Applications 370, Kluwer Academic Publishers Group, Dordrecht, 1996.

[17] W. J. Liu, Q. A Ngô and W. Chen, Ostrowski type inequalities on time scales for double integrals, Acta Appl. Math. 110(1) (2010), 477-497.

[18] W. J. Liu, Q. A. Ngô and W. Chen, On new Ostrowski type inequalities for double integrals on time scales, Dynam. Systems Appl. 19 (2010), 189-198.

[19] W. J. Liu and A. Ngô, A new generalization of Ostrowski type inequality on time scales, An. Stiint. Univ. "Ovidius" Constanta Ser. Mat. 17(2) (2009), 101-114.

[20] W. J. Liu and A. Ngô, An Ostrowski-Grüss type inequality on time scales, Comput. Math. Appl. 58 (2009), 1207-1210.

[21] Q. A. Ngô and W. J. Liu, A sharp Grüss type inequality on time scales and application to the sharp Ostrowski-Grüss inequality, Commun. Math. Anal. 6(2) (2009), 33-41.

[22] E. R. Nwaeze, A new weighted Ostrowski type inequality on arbitrary time scale, Journal of King Saud University 29(2) (2017), 230-234.

[23] A. M. Ostrowski, Über die Absolutabweichung einer Differentiebaren Funktion von ihrem Integralmittelwert, Comment. Math. Helv. 10 (1938), 226-227.

[24] U. M. Özkan and H. Yildirim, Ostrowski type inequality for double integrals on time scales, Acta Appl. Math. 110(1) (2010), 283-288.

[25] A. Tuna and S. Kutukcu, New generalization of the Ostrowski inequality and Ostrowski type inequality for double integrals on time scales, J. Comput. Anal. Appl. 21(6) (2016), 1024-1039.

[26] A. Tuna and D. Daghan, Generalization of Ostrowski and Ostrowski-Grüss type inequalities on time scales, Comput. Math. Appl. 60 (2010), 803-811.

\author{
${ }^{1}$ Department of Mathematics and Computer Science, \\ Alabama State University, \\ Montgomery, AL 36101, USA \\ Email address: skermausour@alasu.edu \\ ${ }^{2}$ Department of Mathematics, \\ Tuskegee UnIVERSITY, \\ Tuskegee, AL 36088, USA \\ Email address: enwaeze@tuskegee.edu \\ *Corresponding Author
}

